Revue d'histoire de l'Amérique française

REYUE D.HISTOIRE DE L'AMÉRIQUE FRANÇAISE

\title{
L'identité professionnelle des infirmières canadiennes-françaises à travers leurs revues (1924-1956)
}

\section{Yolande Cohen et Éric Vaillancourt}

Volume 50, numéro 4, printemps 1997

URI : https://id.erudit.org/iderudit/305601ar

DOI : https://doi.org/10.7202/305601ar

Aller au sommaire du numéro

Éditeur(s)

Institut d'histoire de l'Amérique française

ISSN

0035-2357 (imprimé)

1492-1383 (numérique)

Découvrir la revue

Citer cet article

Cohen, Y. \& Vaillancourt, É. (1997). L’identité professionnelle des infirmières canadiennes-françaises à travers leurs revues (1924-1956). Revue d'histoire de l'Amérique française, 50(4), 537-570. https://doi.org/10.7202/305601ar
Résumé de l'article

L'analyse des deux premières revues des infirmières canadiennes-françaises témoigne des efforts déployés par une élite pour établir un modèle particulier de professionnalisation. Membres du clergé, soeurs hospitalières et médecins souhaitent, en fondant La Veilleuse (1924-1927), contrer l'implantation du modèle laïque de l'infirmière professionnelle canadienne-anglaise. Ils font la promotion d'un modèle apostolique basé sur l'identité féminine et nationale. La création de La Garde-malade canadienne-française (1928-1956) devient toutefois nécessaire devant le désengagement des médecins et la laïcisation croissante de la pratique infirmière. Malgré une plus grande ouverture à l'égard du caractère scientifique des soins infirmiers, on continue de proposer un modèle spécifique de l'infirmière professionnelle canadienne-française jusqu'à la Révolution tranquille.
Tous droits réservés @ Institut d'histoire de l'Amérique française, 1997
Ce document est protégé par la loi sur le droit d'auteur. L'utilisation des services d’Érudit (y compris la reproduction) est assujettie à sa politique d'utilisation que vous pouvez consulter en ligne.

https://apropos.erudit.org/fr/usagers/politique-dutilisation/ 


\title{
L'IDENTITÉ PROFESSIONNELLE DES INFIRMIÈRES CANADIENNES-FRANÇAISES À TRAVERS LEURS REVUES $(1924-1956)^{1}$
}

\author{
YOLANDE COHEN \\ ÉRIC VAILLANCOURT \\ Département d'histoire \\ Université du Québec à Montréal
}

\begin{abstract}
RÉSUMÉ
L'analyse des deux premières revues des infirmières canadiennes-françaises témoigne des efforts déployés par une élite pour établir un modèle particulier de professionnalisation. Membres du clergé, sœurs hospitalières et médecins souhaitent, en fondant La Veilleuse (1924-1927), contrer l'implantation du modèle laïque de l'infirmière professionnelle canadienne-anglaise. Ils font la promotion d'un modèle apostolique basé sur l'identité féminine et nationale. La création de La Garde-malade canadienne-française (1928-1956) devient toutefois nécessaire devant le désengagement des médecins et la laïcisation croissante de la pratique infirmière. Malgré une plus grande ouverture à l'égard du caractère scientifique des soins infirmiers, on continue de proposer un modèle spécifique de l'infirmière professionnelle canadienne-française jusqu'à la Révolution tranquille.
\end{abstract}

\section{ABSTRACT}

The purpose of this article is to study the specific professional model developed by the first French-Canadian nursing publications. Members of the church, nuns and physicians were all trying through La Veilleuse (1924-1927) to establish a model of nursing based on the apostolate of French-Canadian women opposed to the AngloCanadian model. The creation of La Garde-malade canadienne-française (1928-1956) became an obligation with the gradual defection of physicians from the FrenchCanadian model and the progressive secularization of nursing. More open to the scientific character of nursing, the review continued to promote a specific claim of the French-Canadian nurse until the Quiet Revolution.

1. Ce texte présente les résultats partiels d'une recherche subventionnée à la fois par le Conseil de recherches en sciences humaines du Canada et par le Hannah Institute for the History of Medecine. Il est également nécessaire de remercier Louise Bienvenue, Maryann Farkas et les deux évaluateurs anonymes de la Revue d'histoire de l'Amérique française pour leurs judicieuses remarques.

RHAF, vol. 50, n 4, printemps 1997 


\section{INTRODUCTION}

Aborder les questions soulevées par la construction historique de l'identité professionnelle revient souvent à décrire des processus qui semblent inéluctables ou évidents. Ainsi, la réforme des soins infirmiers aboutissant à leur professionnalisation a souvent été perçue comme un processus inévitable accompagnant l'industrialisation et l'urbanisation qu'ont connu la plupart des pays occidentaux au début de ce siècle. La demande accrue de soins et le virage scientifique et technique sont censés expliquer une transformation majeure, qui relègue au rang de vocation de moindre prestige les soins hospitaliers charitables.

Cette vision a été largement nuancée par des études féministes du courant identitaire ${ }^{2}$. On insiste de plus en plus sur l'importance de la vision maternaliste - c'est-à-dire le large mouvement philanthropique faisant la promotion d'idéaux de charité et de dévouement féminins - pour expliquer la contribution des femmes à l'histoire de la santé3.

L'historienne Kathryn McPherson ${ }^{4}$ va dans ce sens. Il est clair selon elle que la professionnalisation des soins infirmiers a permis aux infirmières de se construire un créneau dans le système de santé et de définir leur intervention en termes de science et non plus uniquement en termes de service personnel ou féminin. De plus, elle souligne l'importance de l'organisation et de la discipline tayloristes qui structurent l'ensemble des tâches et la vie des infirmières. Mais elle insiste fortement sur l'intégration par les premières infirmières canadiennes des notions de care et cure dans la pratique quotidienne du nursing et l'intérêt qu'elles portent à construire le plus strictement possible l'ensemble des techniques qui le constituent, tout en y intégrant d'autres paradigmes comme le nurturing, le maternage social des femmes et leur identification à un rôle et à une respectabilité.

Toutefois, son étude des infirmières canadiennes laisse dans l'ombre les infirmières canadiennes-françaises. Bien que ces dernières soient très tôt confrontées au modèle organisationnel de soins infir-

2. Voir notamment Theda Skocpol, Protecting Soldiers and Mothers: the Politics of Social Provision in the United States, 1870s-1920s (Cambridge, Mass, Belknap Press of Harvard University Press, 1992); Seth Koven et Sonya Michel, dir., Mothers of a New World. Maternalist Politics and the Origins of Welfare States (New York, Routledge, 1993).

3. Dianne Dodd et Deborah Gorham, dir., Caring and Curing. Historical Perspectives on Women and Healing in Canada (Ottawa, University of Ottawa Press, 1994).

4. Voir Kathryn McPherson, Bedside Matters. The Transformation of Canadian Nursing, 1900-1990 (Toronto, Oxford University Press, 1996). 
miers décrit par McPherson et implanté au Québec par les Canadiennes anglaises, elles fonctionnent selon des traditions et une organisation des soins différentes ${ }^{5}$. Quelles sont les particularités de l'éthique professionnelle des Canadiennes anglaises et celles de l'éthique de service de leurs consœurs canadiennes-françaises? Y a-t-il un lien à faire entre l'organisation hiérarchique de l'Église et celle du système de Taylor? Les différences, souvent amplifiées par des visions idéologiques, existent, mais doivent être comprises dans le contexte plus large de la conception et de l'organisation des soins.

Lancée au Québec par les infirmières canadiennes-anglaises durant la période $1890-1927^{6}$, la professionnalisation des soins infirmiers a tendance à devenir la référence et le modèle idéologique dominants. Soutenues par des médecins canadiens-anglais, ces infirmières adaptent donc le modèle médical d'organisation aux soins infirmiers: formation et production de savoirs contrôlées par les associations professionnelles d'infirmières, enregistrement légal du titre garantissant l'exclusivité de la pratique, conception hiérarchique et subordonnée des soins infirmiers au pouvoir médical, etc. Les pionnières de la profession sont à l'origine de l'obtention de l'enregistrement partout au Canada. La Loi d'incorporation de l'Association des gardes-malades enregistrées de la province de Québec (AGMEPQ) est adoptée en février 1920. Pour leur part, la majorité des sœurs hospitalières, des infirmières laïques et des médecins canadiens-français y furent d'abord hostiles, n'y voyant qu'ingérence étatique dans des affaires privées et religieuses. Les clivages ethnolinguistiques, qui semblent contrecarrer les ambitions professionnelles des infirmières canadiennes-anglaises, recouvrent-ils des conceptions différentes de la pratique des soins?

À première vue, l'histoire des infirmières canadiennes-françaises, considérée sous l'angle de la professionnalisation, concorderait avec

5. Cette particularité est perceptible à travers les travaux de plusieurs auteurs: Édouard Desjardins, Suzanne Giroux et Eileen C. Flanagan, Histoire de la profession infirmière au Québec (Montréal, Association des infirmières et infirmiers de la province de Québec, 1970); Louise-Hélène Trottier, Évolution de la profession infirmière au Québec de 1920 à 1980, mémoire de maîtrise (sociologie), Université de Montréal, décembre 1982; André Petitat, Les infirmières. De la vocation à la profession (Montréal, Boréal, 1989) et Johanne Daigle, Devenir infirmière: le système d'apprentissage et la formation professionnelle à l'Hôtel-Dieu de Montréal, 1920-1970, thèse de doctorat (histoire), Université du Québec à Montréal, 1990, 2 vol.

6. Yolande Cohen et Louise Bienvenue, «Émergence de l'identité professionnelle chez les infirmières québécoises, 1890-1927», Bulletin canadien d'histoire de la médecine, 11 (1994): 119-151.

7. La question du «retard» historique du Québec est de nouveau d'actualité à la suite de la critique de Ronald Rudin, «La quête d'une société normale: critique de la réinterprétation de l'histoire du Québec», Bulletin d'histoire politique, 3,2 (1995): 9-42 et «Revisionism and the Search for a Normal Society: a Critique of Recent Quebec Historical Writing», Canadian Historical Review, 73 (1992): 30-61. 
l'interprétation du retard général du Québec par rapport au Canada ${ }^{7}$, à condition de considérer le modèle professionnel canadien-anglais comme le seul moderne... Et s'il existait un autre modèle de soins, tout aussi professionnel, chez les Canadiennes françaises? Les effets de mise en discours sont, on le sait, essentiels à l'interprétation adoptée et induisent le caractère moderne ou ancien qu'on attribue à un phénomène. L'étude plus détaillée des processus de professionnalisation des infirmières canadiennes-françaises permet de démonter les mécanismes qui ont conduit à l'adoption d'un modèle. Pour le caractériser, nous chercherons à identifier les bricolages subtils auxquels se sont livrés les différents intervenants dans son élaboration.

L'enjeu que constitue la bataille professionnelle est, on le voit, autant idéologique que programmatique. La confrontation des différentes conceptions sous-jacentes à ces modèles prend toute sa dimension autour de la loi d'enregistrement, car c'est à ce moment que l'un tente de s'imposer à l'autre. Avec l'incorporation de l'AGMEPQ, le discours professionnel discrédite de plus en plus le modèle apostolique canadien-français. C'est ce processus de recouvrement d'un discours par un autre qu'il convient d'analyser. Comment le discours professionnel détrône-t-il celui multiséculaire de la charité? Quels sont les acteurs de cette bataille qui prend les multiples aspects de conflits entre modernité et Ancien Régime, science et charité, care et cure? Les clivages ethnolinguistiques (Canadiennes françaises/Canadiennes anglaises), religieux (sœurs hospitalières/infirmières laïques, clercs/ médecins) et sexuels (hommes/femmes) compliquent davantage l'analyse.

Nous avons choisi de présenter les différentes modalités de construction du discours professionnel, tel qu'il se présente dans les revues professionnelles des infirmières canadiennes-françaises. L'étude de la manière dont les principales intéressées, et surtout leurs élites, conçoivent leur métier et élaborent leurs stratégies pour y donner du sens, permet de mieux comprendre son évolution particulière au Québec et d'éclairer ces débats de façon transversale. Le rôle des revues professionnelles dans l'élaboration et la définition de ces modèles n'est plus à démontrer. Elles furent déterminantes pour l'assise professionnelle des médecins et pour celle des infirmières canadiennes ${ }^{8}$. En favorisant l'émergence d'une élite de la profession et l'unification d'intérêts

8. Voir Éric Vaillancourt, Les rapports médecins-infirmières au Canada et au Québec: analyse de l'interdiscursif à travers leurs revues professionnelles (1867-1920), mémoire de maîtrise (histoire), Université du Québec à Montréal, novembre 1995; Julia Kinnear, "The Professionalization of Canadian Nursing, 1924-1932: Views in the $C N$ and the CMAJ», Bulletin canadien d'histoire de la médecine, 11,1 (1994): 153-174. 
souvent divergents derrière une même bannière professionnelle, les revues sont aussi leurs porte-parole. N'ayant fait l'objet d'aucune étude systématique, la presse des infirmières canadiennes-françaises permet d'observer le mode de recrutement de leur élite et d'analyser les réflexions qu'elle a développées sur le métier.

Deux revues forment le corpus de notre analyse. Quatre ans après l'incorporation de l'AGMEPQ et 19 ans après la fondation de la toute première revue canadienne spécialement destinée aux infirmières ${ }^{9}$, soit en 1924, les sœurs de la Charité de Montréal, communément appelées sœurs grises, mettent sur pied la première revue professionnelle des infirmières canadiennes-françaises. En fait, membres du clergé, sœurs hospitalières, médecins et infirmières laïques se concertent afin d'entretenir une conception chrétienne des soins infirmiers s'accommodant des progrès de la science et des techniques. Toutefois, des questions liées à la professionnalisation - les problèmes du rôle des infirmières et de la formation des diplômées - entraînent le rapide déclin de $L a$ Veilleuse en 1927. Les sœurs grises font appel à une infirmière laïque pour fonder en 1928 une nouvelle revue, La Garde-malade canadienne-française ( $L a G M C F)$. La conception apostolique des soins infirmiers ne sera pas entièrement abandonnée; elle coexistera avec un modèle laïque et nationaliste, axé sur le développement professionnel. À la veille de la Révolution tranquille, la revue cède le pas à une autre, Les Cahiers du nursing canadien, marquant pour un temps la volonté de normalisation et d'intégration des infirmières franco-catholiques dans le giron du nursing canadien.

Nous avons effectué un dépouillement exhaustif de ces revues et analysé tous les articles signés par des infirmières, des médecins et des membres du clergé, et portant sur leur conception de la profession infirmière. Certes, comme dans toute analyse de discours de nature qualitative, l'accent est mis sur les aspects saillants et les propositions nouvelles qui apparaissent au fil des pages. Les limites et attraits de cette méthode, faisant appel à l'intersubjectivité des chercheurs avec leur objet, sont connus. En traquant les reflets de «la conscience d'un Nous ${ }^{10} »$, nous en sommes venus à pouvoir tracer le portrait des prin-

9. C'est l'Association des infirmières du Toronto General Hospital qui met sur pied en 1905 la première revue canadienne spécialement destinée aux infirmières: The Canadian Nurse. La revue se donne dès le départ pour mission de favoriser la solidarité entre toutes les infirmières canadiennes en vue de la reconnaissance officielle de leur profession au moyen de l'enregistrement légal. Yolande Cohen et Louise Bienvenue, loc. cit., 124.

10. La sociologue Andrée Fortin s'exprime ainsi lorsqu'elle cherche à vérifier, par le biais des revues fondées depuis la Conquête, la nature des transformations des rapports des intellectuels québécois avec le social et le politique. Passage de la modernité. Les intellectuels québécois et leurs revues (Sainte-Foy, Presses de l'Université Laval, 1993). 
cipaux intervenants dans ce débat et à trouver la place relative qu'ils occupent dans les revues (proportion des articles publiés, importance et régularité des rubriques, etc.).

$\mathrm{Au}$ terme de cette enquête, les notions de profession, de professionnalisation et de statut professionnel ou d'identité professionnelle seront clarifiées sur les plans sémantique et idéologique. On verra que, pour chacun des protagonistes de la santé, ces notions recouvrent des réalités et des conceptions différentes. Notons toutefois que la terminologie utilisée est celle qui correspond au modèle angloprotestant, où l'utilisation de termes distincts différencie le modèle professionnel (basé sur la conception des soins et du rôle de l'infirmière) du processus de professionnalisation (associé principalement à la création d'écoles et d'associations professionnelles), et du statut professionnel (défini par voie législative). La transformation rapide de ce modèle en un modèle de type organisationnel pousse les élites canadiennes-françaises à se préoccuper du leur. Et même si elles contestent aux anglo-protestants l'antériorité multiséculaire des pratiques apostoliques de soins, il leur faudra définir un modèle et donc se confronter aux autres. Les revues naissent de ce besoin de conceptualisation autant que de la nécessité de maintenir la tradition catholique chez les infirmières.

\section{1 - LA VEILLEUSE (1924-1927) OU LA PÉRENNITÉ D'UNE CONCEPTION APOSTOLIQUE DE L'INFIRMIÈRE CANADIENNE-FRANCAISE}

Au tournant du siècle dernier, plusieurs communautés religieuses ont pris part au mouvement de professionnalisation du système de santé en mettant sur pied les premières écoles d'infirmières ${ }^{11}$. L'instauration des training school of nurses au sein des hôpitaux canadiensanglais les avaient forcées à faire une place aux idéaux de Florence Nightingale. Les médecins, influencés par le haut niveau de compétence acquis par la trained nurse, les poussaient à amorcer cette démarche et à former des sœurs hospitalières et des infirmières laïques plus qualifiées ${ }^{12}$.

La croissance du nombre d'intervenants spécialisés et la présence accrue des infirmières laïques à partir des années 1910 avaient con-

11. Des sœurs hospitalières fondent en 1892 une première école d'infirmières à l'HôtelDieu de Lévis, qui suit de près l'ouverture de la toute première école ouverte au Québec, soit celle du Montreal General Hospital en 1890. Il est à noter que l'école d'infirmières de Lévis ne sera accessible aux candidates laïques qu'en 1944. Édouard Desjardins, et al., op. cit., 111.

12. Le cas des sœurs grises de l'hôpital Notre-Dame de Montréal a été bien étudié. Yolande Cohen, «La contribution des Sœurs de la Charité à la modernisation de l'Hôpital NotreDame de Montréal», Canadian Historical Review, 77,2 (juin 1996): 185-220. 
traint les médecins à des réaménagements ${ }^{13}$. Toutefois, leur hégémonie sur le système des soins n'est vraiment battue en brèche qu'avec l'incorporation de l'AGMEPQ en 1920. Les sœurs hospitalières réalisent que l'État peut désormais accorder un titre professionnel à une pratique que les communautés religieuses exercent par dévotion depuis plusieurs siècles. Bien qu'amendée par les évêques, la Loi de l'assistance publique (1921) inquiète le clergé et les sœurs hospitalières. Même les médecins franco-catholiques, les plus sûrs alliés de l'Église dans la défense du statu quo, se laissent séduire par les bienfaits de l'étatisation et commencent à réclamer des subsides pour le soin aux indigents ${ }^{14}$. S'ils ne peuvent enrayer le mouvement, le clergé et les communautés religieuses, dont l'hégémonie longtemps reconnue sur le secteur des soins apparaît menacée, doivent tenter d'en contrôler les effets. Il s'agit dès lors de signifier de façon explicite, au moyen de la formation et d'une revue, les modalités de développement d'une profession infirmière canadienne-française.

\section{L'instrument du clergé et des scurs hospitalières}

Les médecins de la faculté de médecine de l'Université de Montréal et les sœurs hopitalières s'entendent sur la nécessité d'établir des cours de formation supérieure pour les sœurs hospitalières et les infirmières laïques diplômées durant les étés de 1923 et de $1924^{15}$. Les hospitalières comptent sur les médecins pour acquérir les connaissances nécessaires qui leur permettront de se maintenir dans leur fonction de directrices des écoles d'infirmières, et sur l'université pour leur garantir les diplômes. Elles obtiennent l'appui de $\mathrm{M}^{\mathrm{gr}} \mathrm{J}$ oseph-Vincent Piette, recteur de l'Université de Montréal. Membre éminent du clergé, impliqué dans la naissance de l'Université de Montréal, il collabore régulièrement avec l'archevêque de Montréal, $\mathrm{M}^{\mathrm{gr}}$ Paul Bru-

13. L'étude de l'évolution du nombre d'infirmières diplômées à l'hôpital Notre-Dame de Montréal pour la période 1899 à 1920 nous a révélé qu'il y a une hausse considérable d'élèvesinfirmières laïques à partir de 1910. De 1899 à 1909, 32 religieuses sont formées par l'école contre 16 laïques. Tandis que de 1910 à 1920 , il y a 85 élèves-infirmières laïques pour 18 religieuses. Yolande Cohen, «La contribution des Sœurs de la Charité à la modernisation de l'Hôpital Notre-Dame de Montréal», loc. cit.

14. Dès 1925 , les médecins s'organisent autour de L'Action médicale dans le but d'affirmer une volonté nouvelle de défendre leurs droits, en dépit du discours charitable et apostolique. Maryann Farkas montre bien dans son mémoire l'ambivalence des médecins. Best of Enemies: Quebec Doctors and the State, 1930-1970, mémoire de maîtrise (histoire), Université Concordia, 1987.

15. Ces cours mènent au certificat en direction d'écoles de gardes-malades. La diversité clinique croissante et le développement incessant des techniques médicales rendent nécessaires la formation théorique et universitaire. L'université McGill a ouvert la voie en 1920 en offrant des cours de perfectionnement. André Petitat, op. cit., 182. 
chési et avec l'évêque auxiliaire et administrateur de l'archevêché de Montréal, $\mathrm{M}^{\mathrm{gr}}$ Georges Gauthier. Il suggère la création d'un bulletin d'information pour la promotion des cours de formation supérieure. La filiale des gardes-malades du cours supérieur ${ }^{16}$ reprend cette idée et la publication d'une revue mensuelle devient un de ses objectifs ${ }^{17}$.

Deux sœurs grises, sœur Fafard et sœur Duckett, prennent en charge la première revue professionnelle d'infirmières canadiennesfrançaises au Canada. Formée à l'école d'infirmières de l'hôpital Notre-Dame de Montréal, hôpital affilié à la faculté de médecine de l'Université de Montréal, sœur Fafard a fait des études universitaires en pharmacie à l'université d'Ohio. Elle pratique dans l'Ouest canadien pour devenir plus tard vice-présidente de l'AGMEPQ et directrice générale des hôpitaux des sœurs grises ${ }^{18}$. Quant à sœur Duckett, elle a été directrice d'écoles d'infirmières dans l'Ouest canadien de 1902 à 1909, après sa sortie de l'école de l'hôpital Notre-Dame. Elle revient ensuite à Notre-Dame comme directrice des gardes-malades et tente de convaincre les supérieures générales des communautés de sœurs hospitalières de participer aux cours de formation supérieure. Elle est également à l'origine d'un manuel à l'attention des infirmières qu'on dit être «une réplique des cours donnés par les médecins ${ }^{19}$ ». Jean Browne, présidente de l'Association des infirmières canadiennes, dira d'elles en 1925 qu'elles sont toutes deux les véritables organisatrices des infirmières canadiennes-françaises. Pionnières, ces infirmières éduquées, ces sœurs «progressistes», comme Johanne Daigle les appelle, tentent de faire une synthèse entre l'évolution scientifique et médicale et l'apostolat ${ }^{20}$.

Le premier numéro de La Veilleuse paraît en janvier 1924. Le comité exécutif est composé de $\mathrm{M}^{\mathrm{gr}}$ Piette agissant à titre de contrôleur administratif et du chanoine Émile Chartier, vice-recteur de l'Université de Montréal, comme directeur de la rédaction. La publication de la revue répond au besoin de maintenir une conception chrétienne des soins infirmiers en accord avec les progrès de la science:

16. La filiale est composée de quatre sœurs et d'une laïque, la trésorière, Anasie Deland. Il y a également sept conseillères, dont une laïque, Rose McCaffrey.

17. La filiale a quatre objectifs: $1^{\circ}$ assurer un lien entre l'université et la profession; $2^{\circ}$ travailler à la conception de cours post-scolaires pour les infirmières diplômées; $3^{\circ}$ organiser un congrès annuel pour les infirmières franco-catholiques; $4^{\circ}$ publier La Veilleuse. «Notes pour l'histoire de la profession», La Veilleuse, 1,11 (novembre 1924): 7.

18. Édouard Desjardins, et al., op. cit., 231.

19. Ibid., 224.

20. Johanne Daigle, op. cit., 136. 
Que dit ce premier numéro du premier organe consacré, dans le Canada français et catholique, à la profession dont elle s'honore de faire partie? En quelques pages [...] il raconte les dévouements que le sens catholique a inspirés aux gardes du passé. Il lui enseigne à ne pas briser cette longue chaîne, en lui prouvant la noblesse du but vers lequel elle tend elle-même. D'autres pages lui dévoilent le champ nouveau que l'expérience ouvre à son zèle. Des informations la renseignent sur l'efficacité des traitements récents, sur les procédés encore peu répandus malgré leur réelle utilité ${ }^{21}$.

S'il y a ouverture aux progrès de la science, elle ne se fait que dans la continuité d'une tradition catholique qui permet seule de «maintenir, relever même, le niveau de la profession ${ }^{22}$ » des infirmières canadiennes-françaises. En fait, la revue a pour objectif essentiel d'élaborer et de formaliser un modèle catholique d'infirmière professionnelle canadienne-française.

\section{Une conception chrétienne des soins infirmiers canadiens-français}

La Veilleuse publie notamment les leçons sur la garde-malade à travers les âges, dispensées par l'abbé O.-P. Gauthier au cours de formation supérieure de juillet 1923. Il faut, dit-il «donner dans nos écoles une note franchement chrétienne à l'enseignement de l'histoire professionnelle ${ }^{23}$ », car l'on doit se méfier des manuels écrits par les médecins et les infirmières laïques au sujet de l'histoire de la profession infirmière: «Tous ceux que l'on connaît sont écrits par des médecins ou des 'gardes' non catholiques et l'on y rencontre quantité de remarques et d'affirmations inexactes, fausses et parfois absolument injustes ${ }^{24}$.» Il critique le modèle de Florence Nightingale, source du débat:

Peut-être aussi accepte-t-on trop facilement de suivre les écoles non catholiques et de s'en tenir à Mlle Nightingale comme au nec plus ultra de la garde-malade [...] Certes, on doit reconnaître les mérites incontestables de cette remarquable réformatrice; mais quand on possède à son actif, dans l'histoire des services rendus à l'humanité souffrante, tout le glorieux passé de l'Église catholique, vraiment il semble qu'il n'est pas difficile de chercher plus haut et de trouver mieux, comme inspiratrice de toutes les qualités que nous voulons voir chez 'nos' gardes-malades ${ }^{25}$.

21. Chanoine Émile Chartier, «En veillant», La Veilleuse, 1,1 (janvier 1924): 2.

22. Ibid., 3.

23. Abbé O.-P. Gauthier, «Notes pour l'histoire de la profession», La Veilleuse, 1,1 (janvier 1924): 14.

24. Ibid.

25. Ibid., 15. 
Dans l'esprit des membres du clergé, la foi catholique doit occuper une place prédominante dans les soins et ce, même lorsque l'on reconnaît l'efficacité de la science moderne: «Â côté de la science professionnelle mettons une instruction religieuse solide [de toute façon] la médecine vient de Dieu ${ }^{26} . »$ En janvier 1925, dans un article intitulé $L a$ vocation de la garde-malade, le père $\mathrm{A}$. Lamarche démontre la dimension chrétienne du travail de l'infirmière où des «vertus innombrables: ponctualité, dévouement, courage, fermeté, douceur, patience et prudence doivent être fondues ensemble, en une sorte de cimentation intérieure qui a nom la charité, puis éclairées d'une lumière souveraine qui est précisément l'esprit de foi ${ }^{27}$ ».

Le vocable garde-malade pour désigner l'infirmière professionnelle reflète l'importance de cette conception hautement chrétienne des soins infirmiers ${ }^{28}$. Chaque livraison de La Veilleuse comporte au moins deux articles insistant sur la dimension apostolique de la pratique infirmière et rappelle l'héritage catholique de la nouvelle profession dont seule une femme peut s'acquitter efficacement.

La femme est perçue dans La Veilleuse comme étant un être pur et naïf dont on doit faire l'éducation; c'est l'unique façon d'en faire une bonne mère de famille. Le père Henri Garrouteigt, aumônier de l'Hôtel-Dieu de Montréal, affirme en juillet 1924 que l'infirmière professionnelle doit être dotée d'une instruction religieuse solide afin qu'elle soit informée «de ce qu'il faut penser de certaines questions délicates auxquelles son état la mêle ${ }^{29} \gg$.

Les membres du clergé tiennent toutefois en haute estime le médecin. Ce dernier demeure une figure d'autorité à qui l'infirmière doit le plus grand respect. Le père Lamarche emploie même cette formule lapidaire: «C'est avant tout au médecin qu'il appartient de parler de la garde-malade aux gardes-malades ${ }^{30} . »$

\section{L'ambiguïté du discours des médecins}

Les découvertes de Pasteur, la bactériologie et l'hygiène, de même que la création des laboratoires, les progrès de la clinique, de la thérapeutique et de la standardisation ont fait du médecin un acteur es-

26. Père Henri Garrouteigt, «L'étude», La Veilleuse, 1,7 (juillet 1924): 3.

27. Père A. Lamarche, "La vocation de garde-malade», La Veilleuse, 2,1 (janvier 1925): 3 .

28. André Petitat traite efficacement de cette question, op. cit., 53 et 70 . Nous reviendrons plus loin sur cette dimension.

29. Père Henri Garrouteigt, «L'étude», La Veilleuse, loc. cit.

30. Père A. Lamarche, «La vocation de garde-malade», La Veilleuse, loc. cit., 2. 
sentiel du système de santé ${ }^{31}$. Les membres du clergé et les sœurs hospitalières reconnaissent le rôle primordial joué par la profession médicale lorsqu'il est question de la formation des infirmières.

La collaboration des médecins à La Veilleuse prend surtout la forme de la reproduction de notes de cours expliquant la matière médicale aux infirmières. Les médecins enseignent la chimie, le cancer, le pouls des patients, l'hygiène, les réalisations de Pasteur, etc. Mais ils traitent aussi de l'éthique professionnelle des infirmières et des lois régissant la profession. De ces textes émerge une conception chrétienne des soins infirmiers très proche de celle véhiculée par les membres du clergé et par les sœurs hospitalières, et qui se juxtapose aux enseignements plus techniques qu'ils diffusent.

En mars 1924, La Veilleuse reprend le discours du docteur E.-P. Benoit ${ }^{32}$, médecin à l'hôpital Notre-Dame et professeur de clinique médicale de l'Université de Montréal, prononcé à l'automne 1923 pour les hospitalières de Saint-Joseph de l'Hôtel-Dieu de Montréal ayant suivi le cours de formation supérieure. Le docteur Benoit est un personnage incontournable lorsqu'il est question de la professionnalisation des infirmières du Québec. Le niveau de compétence des infirmières ne le laisse pas indifférent. Il publie même un manuel de matière médicale à l'usage des gardes-malades dès $1904^{33}$. Traçant un bref portrait historique de la fondation de l'Hôtel-Dieu de Montréal, il reconnaît le modernisme des sœurs hospitalières et louange leurs efforts: «Si vous êtes une communauté cloîtrée, vous n'avez pas voulu fermer les yeux sur ce qui se passe en dehors de vos murs [...] Vous voulez bien vous adapter aux besoins nouveaux qui se font sentir $[\ldots]^{34}$ »

Il termine en lançant un appel au renouveau catholique:

31. Voir Peter Keating et Othmar Keel, Santé et société au Québec: XIX ${ }^{e}-X X^{e}$ siècles (Montréal, Boréal, 1995).

32. Le docteur Benoit est notamment président de la commission chargée d'étudier les questions relatives aux cours de gardes-malades. Acteur de premier plan en ce qui concerne le développement de la santé publique au Québec, ce fils de bourgeois né le 24 décembre 1869 devient rédacteur en chef de L'Union médicale du Canada en 1895, trois ans seulement après avoir obtenu son diplôme de l'Université Laval. Il sera président de la Commission des gardesmalades rattachée à la faculté de médecine de l'Université de Montréal jusqu'à son décès en 1946.

33. C'est la préparation du cours matière médicale pour les élèves-infirmières de l'hôpital Notre-Dame qui l'amena à synthétiser les notions relatives à l'administration des médicaments aux malades. Emmanuel-Persillier Benoit, Manuel de matière médicale à l'usage des gardesmalades (Montréal, Librairie Beauchemin, 1904).

34. E.-P. Benoit, «Méditation historique», La Veilleuse, 1,3 (mars 1924): 10. 
Efforçons-nous maintenant d'établir le renouveau catholique par l'exemple canadien; notre race et notre religion n'en seront que plus respectées en Amérique, l'œuvre universitaire qui nous est si chère n'en sera que plus puissante. Mais sachons aussi, et c'est très important pour notre unité nationale, demeurer fidèles à nos traditions ${ }^{35}$.

Comme les membres du clergé qui s'expriment dans la revue, les médecins privilégient un discours à double entrée. Mais si les membres du clergé conçoivent que l'infirmière doit être d'abord investie d'une mission divine et qu'elle doit, pour accomplir cette mission, être formée scientifiquement, tout le problème réside pour les médecins dans la priorité à accorder à l'un ou l'autre de ces deux attributs. L'important est de pouvoir compter sur une assistante compétente qui sait se tenir. Dans les années 1920, l'incontournable sœur hospitalière correspond encore le mieux à cette définition.

La Veilleuse publie en juin 1924 le discours prononcé par le docteur C.-A. Bouchard, chirurgien de l'hôpital Saint-Joseph de TroisRivières, lors d'une remise de diplômes à cinq infirmières laïques. Il précise que «Florence Nightingale n'a pas inventé le soin des mala$\operatorname{des}^{36} »$. Elle a plutôt réussi à réformer une pratique ancestrale grâce à son «esprit pratique, avide de nouveautés, ennemi de la routine, discipliné et méthodique ${ }^{37}$ ». S'il faut suivre son exemple pour assurer, leur dit-il, «le succès matériel de vos œuvres ${ }^{38}$ », il faut également dépasser ces préoccupations matérielles en faisant preuve d'un «véritable dévouement ${ }^{39}$ », un dévouement d'ascète à l'image des sœurs de la Charité qui accompagnèrent Nightingale en Crimée.

Cependant, la formation scientifique s'ajoute nécessairement à la mission apostolique, et c'est ce qui distingue l'infirmière professionnelle de la soignante. La Veilleuse publie le discours prononcé par le docteur Benoit au premier congrès canadien-français des gardes-mala$\operatorname{des}^{40}$ :

[...] il faut bien admettre que les gardes-malades de cette époque [XVII ${ }^{\mathrm{e}}$ siècle] ne constituaient pas une profession, et que leur vocation leur tenait lieu de diplôme. [Mais en 1924], pour le soin

35. Ibid. 1924): 11

36. C.-A. Bouchard, «À l'hôpital St-Joseph des Trois-Rivières», La Veilleuse, 1,6 (juin

37. Ibid

38. Ibid., 12 .

39. Ibid., 13

40. Le premier congrès des infirmières professionnelles canadiennes-françaises a lieu à Montréal les 8 et 9 septembre 1924. 
efficace des malades, la vocation seule, n'est pas suffisante [...] C'est par l'instruction, c'est-à-dire la science, que l'on acquiert le complément nécessaire de la vocation. Aujourd'hui la formation scientifique est une obligation pour la garde-malade ${ }^{41}$.

Il avait noté précédemment l'importance de cette nouvelle qualification pour exercer «[...] sa profession dans toutes ces spécialités. [...] Grâce au développement de la science médicale, la société moderne réserve une place excellente à la garde-malade dans les professions que l'on appelle libérales ${ }^{42}$ ». Pourtant, même s'il soutient que l'infirmière est «la collaboratrice scientifique du médecin ${ }^{43}$ », le docteur Benoit ne considère pas l'infirmière comme une professionnelle à part entière et indique qu'il y a différents statuts attachés à chaque profession.

Les développements de l'hygiène permettent d'ajouter un élément à ce scénario. La collaboratrice scientifique du médecin est amenée à jouer un rôle éducatif tout à fait différent de ce qu'a pu être celui de la sœur hospitalière. C'est à ce moment que l'infirmière laïque se démarque de la sœur hospitalière. Elle peut de plus s'acquitter de certaines tâches interdites aux religieuses, en obstétrique par exemple. Benoit pense donc aux infirmières-hygiénistes quand il ouvre la perspective d'une profession libérale aux infirmières. Le docteur J.-A. Baudouin $^{44}$ va également dans ce sens en préconisant l'intervention éclairée des infirmières dans l'adoption de nouvelles habitudes de vie et des mesures élémentaires d'hygiène par les populations:

Par ses explications répétées, par ses démonstrations faites à domicile, l'infirmière peut ainsi changer la mentalité de nos gens trop imbue de préjugés. Elle s'est démontrée, non seulement à l'étranger mais aussi chez nous, l'éducatrice la plus écoutée des familles. Son rôle est donc des plus importants dans tous les domaines de l'hygiène ${ }^{45}$.

Mais en dehors de cette catégorie, qui échappera de plus en plus au contrôle des médecins, ces derniers demeurent vagues et personnels pour caractériser le rôle des infirmières. Seule la question de la forma-

41. E.-P. Benoit, Notes pour l'histoire de la profession, La Veilleuse, 1,10 (octobre 1924): 5 et 6.

42.. Ibid., 2 et 3.

43. Ibid., 6 .

44. Le docteur Joseph-Albert Beaudoin est alors un hygiéniste convaincu qui se consacre entièrement depuis 1920 au Conseil dupérieur d'hygiène de la province de Québec. Voir Georges Desrosiers, Joseph-Albert Beaudoin (1875-1962): professeur d'hygiène, Bulletin canadien d'histoire de la médecine, 10 (1993): 251-268.

45. J.-A. Beaudoin, La coupe commune, La Veilleuse, 1,7 (juillet 1924): 13. 
tion des infirmières est perçue par les médecins comme un enjeu majeur. Par elle, on entrevoit la réflexion des uns et des autres au sujet du rôle des infirmières. C'est ce qui mobilise certains médecins et les pousse à obtenir en 1922 le premier amendement à la Loi d'incorporation de l'AGMEPQ. Plusieurs articles de la loi de 1920, en particulier ceux qui attribuent aux associations d'infirmières la responsabilité de la collation des grades et ceux qui stipulent que les infirmières des hôpitaux généraux de moins de 50 lits doivent nécessairement fréquenter plus d'une école pendant leurs trois années de formation, sont perçues comme des entraves à leur pouvoir. Emboîtant soudain le pas aux 65 infirmières laïques qui avaient fait circuler une pétition afin que les infirmières puissent être enregistrées après un cours de deux ans, des médecins de la faculté de médecine de l'Université de Montréal proposent de prendre en charge l'enseignement dispensé aux élèves-infirmières. L'amendement adopté en mars 1922 répond favorablement à cette double revendication: deux ans de formation suffiront aux élèves-infirmières d'une école reconnue par la faculté de médecine de l'Université de Montréal pour être enregistrées. L'AGMEPQ perd le monopole de la collation des diplômes et celui de la formation. Au Québec, le cadre professionnel mis en place par les associations d'infirmières canadiennes-anglaises vient de s'effriter. Cela contribuera à accentuer le clivage entre les pratiques et les formations des deux communautés anglo-protestante et franco-catholique.

Deux ans plus tard, en avril 1924, la direction de La Veilleuse publie la note que le docteur Benoit, président du comité des gardesmalades, leur fait parvenir. Elle a pour objet l'annonce de la tenue d'un examen universitaire obligatoire et uniforme pour toutes les écoles d'infirmières de la province à partir de 1925. Il s'agira en fait du deuxième amendement à la Loi d'incorporation de l'AGMEPQ qui annonce la mise en place d'un bureau conjoint d'examinateurs formé de médecins de chaque université de la province et de trois membres de l'AGMEPQ. Ce bureau aura le mandat de faire subir les examens aux aspirantes qui ont suivi leur formation dans des hôpitaux affiliés aux universités ${ }^{46}$. Les médecins s'assurent que les infirmières seront formées selon leurs exigences et qu'elles seront ainsi dignes du titre de collaboratrice scientifique. Sous couvert d'offrir des garanties au public, il s'opère une lente révision des modalités de formation qui répond en grande partie aux exigences des médecins d'uniformiser les cours de formation et de transférer entièrement la collation des grades aux institutions universitaires. Cette mesure relègue au second plan le

46. Pour plus de renseignements, voir Yolande Cohen et Louise Bienvenue, loc. cit. 
rôle des associations professionnelles d'infirmières qui étaient le fer de lance de la loi d'enregistrement de 1920.

Ainsi, l'ambiguité du discours des médecins traduit en réalité un amenuisement de leur appui au modèle apostolique de l'infirmière. Si ce modèle continue d'alimenter leur discours en faveur d'une collaboratrice, la responsabilité de sa formation fait pencher la balance du côté d'une professionnelle. Car une fois réglé le problème de la formation, les médecins se désintéressent de la question. Dès lors privés de leur appui, le clergé et les sœurs hospitalières voient leurs efforts minés. Il faut voir là un des éléments qui conduiront à l'échec de $L a$ Veilleuse.

Ce n'est pas du coté des infirmières laïques, encore minoritaires, qu'il faut attendre un renouveau. Leur implication demeure secondaire et elles se contentent d'intervenir au sujet de l'application de certaines techniques: consultation pour nourrissons, exécution de massages, établissement de la fiche scolaire des enfants examinés, etc. Et lorsqu'elles ont l'occasion de réfléchir sur les fondements de leur profession, elles font état d'une conception des soins infirmiers très similaire à celle des membres du clergé et des sœurs hospitalières.

La Veilleuse ne survivra pas au désengagement progressif des médecins, aux problèmes financiers ${ }^{47}$, au décès de sœur Fafard en 1925 et au transfert de sœur Duckett à Winnipeg en $1927^{48}$. La publication erratique de la revue à partir de juillet 1926 illustre son essoufflement ${ }^{49}$. Une direction laïque relèvera-t-elle les défis? En mai 1927, sœur Duckett demande explicitement à Charlotte Tassé d'en prendre la direction: «C'est le désir de nos hospitalières que vous preniez l'initiative de la réorganisation de La Veilleuse. Vous comprenez que la revue des gardes-malades doit passer sous une tutelle autre que celle d'une communauté religieuse ${ }^{50}$.» La fin de La Veilleuse est annoncée

47. La revue n'est pas subventionnée et ne peut compter que sur la vente d'espaces publicitaires et d'abonnements. Les sœurs hospitalières et les infirmières laïques sont-elles peu intéressées ou manquent-elles tout simplement de temps pour collaborer activement et s'abonner? Quoi qu'il en soit, on lance de nombreux appels et on cherche par tous les moyens à augmenter la liste des abonnées.

48. Estelle Mitchell explique que le Conseil général des sœurs grises envoie sœur Duckett et sœur Despins à Gravelbourg en Saskatchewan pour y fonder un hôpital. Elles sont sur place dès le 2 juillet 1927 alors que les travaux de construction débutent. Voir Estelle Mitchell, Les Sours Grises de Montréal à Rivière-Rouge, 1884-1984 (Montréal, Éditions du Méridien, 1987), 223-224.

49. Les numéros de juillet et d'août 1926 et ceux de juillet, août et septembre 1927 ne sont pas publiés.

50. Lettre du 23 mai 1927 de sœur Duckett à Charlotte Tassé dans Albertine FerlandAngers, L'École d'infirmières de l'Hôpital Notre-Dame, 1898-1948 (Montréal, Éditions Contrecœur, 1948), 58. 
en septembre 1927, à la suite «de difficultés et de circonstances incontrôlables ${ }^{51} \gg$.

\section{2 - LA GARDE-MALADE CANADIENNE-FRANÇAISE (1928-1956) OU L'ABANDON GRADUEL D'UNE CONCEPTION PROPRE DE L'INFIRMIÈRE CANADIENNE-FRANCAISE}

À la réunion qui doit décider de la structure de la nouvelle revue, à l'Université de Montréal le 10 décembre 1927, le docteur Télesphore Parizeau, vice-doyen de la faculté de médecine, appuyé par $\mathbf{M}^{\mathrm{gr}}$ Piette et le docteur Benoit, proposent d'intituler la revue La Garde-malade canadienne-française. Le docteur Benoit, aussitôt élu rédacteur en chef, exprime le souhait que la revue soit placée sous les patronnages de $M^{\text {gr }}$ Gauthier, $M^{\text {gr }}$ Piette et du docteur Louis de LotbinièreHarwood, professeur de gynécologie ${ }^{52}$.

Edith B. Hurley, professeure à l'école d'hygiène sociale appliquée de l'Université de Montréal, suggère pour sa part que des sœurs hospitalières soient membres de l'exécutif de la nouvelle revue. Celles-ci refusent car, disent-elles, «nos règlements de vie religieuse sont in-

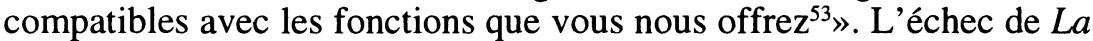
Veilleuse a rendu les sœurs conscientes de l'importance pour elles de changer de stratégie. Elles souhaitent que l'exécutif soit composé d'infirmières laïques. D'ailleurs, elles ont demandé à Charlotte Tassé, la surintendante laïque de l'école d'infirmières du sanatorium Prévost ${ }^{54}$, de diriger la nouvelle revue. Le premier comité exécutif de la nouvelle revue comprendra effectivement une majorité d'infirmières laïques: outre le docteur E.-P. Benoit au poste de rédacteur en chef et Charlotte Tassé comme directrice, Rachel Tassé ${ }^{55}$ est secrétaire-trésorière, Edith B. Hurley et Marie Pelletier, infirmière-hygiéniste, sont conseillères.

51. La Garde-malade canadienne-française, 1,1 (janvier 1928): 26. À l'avenir, La GMCF.

52. $\quad \mathrm{M}^{\mathrm{gr}}$ Gauthier est chancelier de l'Université de Montréal et $\mathrm{M}^{\mathrm{gr}}$ Piette en est toujours le recteur. Quant au docteur Harwood, il est le doyen de la faculté de médecine depuis 1918, à la suite du décès du docteur Emmanuel-Persillier Lachapelle.

53. Rachel Tassé, «Fondation de la revue», $L a$ GMCF, 1,1 (janvier 1928): 30.

54. Le sanatorium Prévost est une institution de soins fondée en juillet 1919 par le docteur Albert Prévost afin de traiter les cas de maladies nerveuses. L'institution comprend dès le début une école d'infirmières dirigée par Tassé.

55. Nous ne savons pas s'il existe un lien de parenté entre Charlotte et Rachel Tassé. Nous savons seulement que Rachel Tassé quitte son poste en 1932 et qu'elle est remplacée par Suzette Panet-Raymond. Cette dernière est diplômée de l'école d'infirmières de l'hôpital SainteJustine. Elle a travaillé dans le service privé au cours des années 1920 pour ensuite entrer au service de l'hôpital Sainte-Justine dans les années 1930 jusqu'à sa retraite en 1964. «Mademoiselle Rachel Tassé», La GMCF, 5,9 (septembre 1932): 464. 
Quant aux sœurs hospitalières, on les retrouve au comité général d'administration qui conseille le comité exécutif. Ce comité consultatif élargi se réunit une ou deux fois par an «pour discuter des affaires de la revue et de la profession ${ }^{56} \%$. En 1928, il se compose de cinq médecins, de six religieuses et de sept infirmières laïques ${ }^{57}$. Quatre ans plus tard, sur les dix membres, on compte cinq religieuses et quatre infirmières laïques agissant sous la présidence du docteur Benoit. Toutefois à partir de mai 1938, aucune religieuse ne fait partie du comité général, tandis que la composition de l'exécutif reste sensiblement la même ${ }^{58}$.

Si l'on sait par ailleurs que les religieuses gardent encore un large contrôle sur la formation des infirmières et la direction des soins infirmiers dans plusieurs hôpitaux de la province, leur départ des instances de direction de la revue, qui ressemble plus à une mise à l'écart, soulève bien des questions. Pensent-elles qu'elles ont un rôle plus important à jouer ailleurs? L'Église est-elle prête à sacrifier leurs prérogatives au profit du maintien de l'orientation générale dans un milieu qu'elle veut influencer autrement? Le discours apostolique est-il devenu insoutenable dans une revue professionnelle?

De fait, tous semblent redécouvrir l'importance des infirmières laïques, diplômées et étudiantes, à qui était initialement destinée la revue. On se rappelle que dans le programme annoncé par Charlotte Tassé, elles étaient directement visées: «[...] vous assurerez l'existence de la revue et, peut-être bien, ajouterez-vous un peu plus d'influence, d'autorité et de considération au corps professionnel dont vous faites partie et dont vous représentez le prestige et l'honneur ${ }^{59}$.»

La GMCF doit être leur porte-parole et leur permettre d'exprimer leurs opinions en demeurant à l'affût des développements scientifiques et des innovations technologiques. Tassé mise beaucoup sur l'im-

56. Rachel Tassé, «Fondation de la revue», $L a G M C F$, loc. cit.

57. Il se compose alors des docteurs Télesphore Parizeau, vice-doyen et directeur des études à la faculté de médecine de l'Université de Montréal, E.-P. Benoit, E.-A. René de Cotret, J.-E. Dubé et Oscar Mercier. Du côté des sœurs hospitalières, il y a sœur Allard de l'Hôtel-Dieu de Montréal, sœur Robert de l'hôpital Notre-Dame, sœur Augustine de l'hôpital Saint-Jean-deDieu, sœur Saint-Tiburce de l'hôpital de la Miséricorde, sœur Valérie de la Sagesse de l'hôpital Sainte-Justine et sœur Marie-de-Lourdes de l'hôpital Sainte-Jeanne-d'Arc. En ce qui concerne les infirmières laïques, on retrouve les trois mêmes qu'au comité exécutif en plus d'Odile Dupuis, surintendante des infirmières au service de l'hygiène de l'enfance de la Ville de Montréal, Angeline Michaud, directrice du service social de l'Institut Bruchési, et Emma Rocques, de l'Assurance-Vie Métropolitaine.

58. Le rédacteur en chef est toujours le docteur Benoit et la directrice demeure Charlotte Tassé. Bernadette Lépine est maintenant la secrétaire-trésorière et les deux conseillères sont mesdames Arthur Deschênes et Suzette Panet-Raymond.

59. Charlotte Tassé, «Notre Programme», La GMCF, 1,1 (janvier 1928): 5. 
pact professionnel de la revue et sur le rayonnement qu'elle pourrait avoir en tant qu'organe des infirmières canadiennes-françaises: «Elle espère intéresser également les gardes-malades faisant du service hospitalier ou institutionnel, comme celles qui déploient leur activité sur le vaste champ du service privé ou social ${ }^{60} . »$

Sept ans après la fondation de $L a G M C F$, le tournant est réellement amorcé: «N'oublions pas que la G.M.C.F. est le seul journal français du genre imprimé au Canada. Il est le lien qui unit les écoles; il est surtout l'agent de diffusion de nos opinions, que nous devons faire connaître et faire respecter ${ }^{61}$.» Le rappel de son identité lui permet de se distinguer des autres revues: l'attachement à la langue française est très souvent mis en évidence et l'idéologie de la famille comme fondement de la survie du fait français en Amérique est primordiale. Ce qui n'empêchera pas $L a G M C F$ de correspondre avec 23 revues à travers le monde et d'inscrire, au nom de l'avancement de la profession infirmière, la perspective nationale dans un espace international qui n'est plus uniquement catholique mais professionnel. Ainsi s'ouvrent de nouvelles avenues de développement qui impliquent une redéfinition du modèle proposé par la revue.

\section{Vers une conception lä̈que des soins infirmiers}

Le ton de plusieurs articles comme leur contenu laissent à penser que le modèle défendu par $L a G M F C$ est sensiblement le même que celui de La Veilleuse. Les collaboratrices laïques de La GMCF mettent l'accent sur la dimension apostolique, charitable et féminine de la pratique infirmière.

Cette conception traditionnelle des soins infirmiers reste présente dans la revue jusqu'en 1956. Encore en février 1942, la Société d'infirmières missionnaires, fondée à Montréal par des infirmières laïques désireuses de servir Dieu ${ }^{62}$, annonce dans les pages de la revue qu'elles «s'engagent en vertu de leur service de fidélité à travailler à la gloire de Dieu et au salut des âmes dans les missions qui deviendront leur champ d'apostolat ${ }^{63}$ ». Tassé fait elle-même la promotion du mouvement missionnaire laïque, mouvement par lequel «nous aug-

60. Ibid.

61. La GMCF, 8,11 (novembre 1935): 649 .

62. À noter aussi la fondation en 1935 de l'Association catholique des infirmières canadiennes. La direction de l'association, qui est officiellement autorisée par le gouvernement du Québec en février 1936, se compose de 10 sœurs hospitalières et de 10 infirmières laïques. Au cours de la même année, le regroupement compte 690 membres actifs (des laïques et des sœurs hospitalières). Mlle Cantin, «Rapport de l'Association catholique des infirmières canadiennes», La GMCF, 9,8 (août 1936): 347-349.

63. «La Société des infirmières missionnaires», La GMCF, 19,8 (août 1946): 361. 
menterons en nous les idées de renoncement, d'abnégation et de dévouement ${ }^{64}{ }^{\prime}$.

Or, nous pouvons constater l'émergence à partir de la fin des années 1920 d'un discours parallèle, plus combattif, invitant les infirmières à s'unir et à contrôler leur profession. Le modèle professionnel des infirmières canadiennes-anglaises est toujours cité en exemple ${ }^{65}$ et Charlotte Tassé suggère même de faire du rattrapage: «C'est le vœu que, toutes, nous devons exprimer, si nous voulons conserver le respect et l'estime de nos compagnes anglaises; croyons-le, le meilleur moyen d'y arriver, c'est encore de prouver à tous que, bientôt, nous n'aurons rien à leur envier $[\ldots]^{66} . »$

Les élèves-infirmières sont également incitées à former leurs propres associations, sans l'intervention des sœurs hospitalières. L'influence décisive est celle du mouvement international des infirmières. En décembre 1937, La GMCF publie notamment l'allocution prononcée par Grace M. Fairley du Montreal General Hospital au congrès international des infirmières à Londres. Première présidente de la Graduate Nurses Association of the Province of Quebec de 1917 à 1918, avant de collaborer activement à la fondation de l'AGMEPQ, elle évoque les raisons qui favorisaient la formation d'associations dirigées par les élèves-infirmières:

La plupart des administrateurs d'hôpitaux et des infirmières chargées de l'instruction des élèves étaient favorables à ce mouvement; ils se rendaient compte qu'on préparait ces jeunes filles à accepter des responsabilités et qu'elles en seraient d'autant plus capables si elles avaient l'occasion, au cours de leurs études, de conduire leurs propres affaires ${ }^{67}$.

Ces quelques exhortations à plus d'autonomie deviennent systématiques à partir de la publication du Rapport Weir en 1932. Les sœurs hospitalières, qui administrent et dirigent encore la plupart des 105.

64. Charlotte Tassé, «Le mouvement missionnaire laïque», La GMCF, 14,3 (mars 1941):

65. Il est tout de même intéressant de sentir la forte influence du modèle canadienanglais. Bien qu'une infirmière sur deux soit canadienne-française, l'impact du modèle canadienanglais semble très important. Par exemple, en 1941, il y a 4618 membres de l'AGMEPQ comparativement à 3240 en 1935. Mais en réalité seulement 4167 ont payé leur cotisation et sont en règle. De ce nombre, 2117 sont des Canadiennes françaises et 2050 des Canadiennes anglaises. «Rapports présentés à l'assemblée annuelle de l'association des gardes-malades enregistrées de la province de Québec», La GMCF, 14,5 (mai 1941): 235.

66. Charlotte Tassé, "Une visite à travers la province», $L a$ GMCF, 1,10 (octobre 1928): 7.

67. «Congrès de Londres-Association d'élèves dans les écoles d'infirmières», La GMCF, 10,12 (décembre 1937): 555. 
écoles d'infirmières, et les médecins, qui contrôlent l'accès à la profession, sont fréquemment critiqués. En décembre 1934, la direction affirme:

On a remarqué sans doute que la collaboration des infirmières fut plus variée, plus fréquente et plus substantielle que par les années passées [...] Nous ne voulons, certes pas affirmer que la collaboration médicale nous soit indifférente! Nous avons simplement souhaité que dans l'ensemble, la revue soit rédigée par des infirmières $^{68}$.

Élément déclencheur du mouvement d'émancipation des infirmières laïques, l'enquête du docteur Weir ${ }^{69}$ s'amorce en novembre 1929 afin de proposer des recommandations en vue d'améliorer la formation des infirmières. Financée au tiers par la Canadian Medical Association et aux deux tiers par l'Association canadienne des infirmières, l'enquête est effectuée à travers le pays. Elle se termine le 31 août 1931 après la visite de plus de 145 écoles d'infirmières et l'organisation de 650 colloques avec des infirmières, des étudiantes, des médecins et des représentantes du public. Le docteur Weir dénonce, entre autres, le temps précieux que les infirmières perdent à accomplir diverses tâches ménagères: elles passent plus de $75 \%$ de leur temps à balayer, polir, épousseter, nettoyer et désinfecter les murs, meubles, matelas et oreillers ${ }^{70}$.

Claire Godbout de l'Hôtel-Dieu de Montréal fait, en janvier 1933, le compte rendu du résumé de cette enquête effectué par le docteur Bazin $^{71}$ : «Le Résumé éclaire le problème d'une évidence: l'enquête veut servir à l'élaboration d'un programme d'enseignement susceptible d'améliorer les services et la profession de garde-malade au Canada $^{72} . »$ Godbout souligne qu'il faut remettre en question le caractère apostolique de la profession qui a tendance à se consolider en période de crise: «[...] un noble passé n'implique pas une forme définitive de dévouement mais devrait plutôt inspirer des activités toujours conformes aux besoins de l'heure ${ }^{73}$.» De nouvelles exigences éducatives sont

68. «Souhaits de fin d'année», La GMCF, 7,12 (décembre 1934): 713.

69. Le docteur G. M. Weir est le directeur du département d'éducation de l'université de la Colombie britannique lorsqu'on lui confie la responsabilité de mener l'enquête.

70. G. M. Weir, Survey of Nursing Education in Canada (Toronto, University of Toronto Press, 1932), 191-193.

71. Le docteur Bazin publie un résumé de l'enquête du docteur Weir alors que le docteur Baudouin en fait la traduction. On invitera d'ailleurs les infirmières canadiennes-françaises à se prémunir de l'ouvrage «précieux et important» du docteur Baudouin. «La traduction du livre du Docteur Weirn, La GMCF, 6,3 (mars 1933): 170.

72. Claire Godbout, «Résumé de l'enquête du Dr. G. M. Weir au sujet de l'état du 'Nursing' au Canada», $L a$ GMCF, 6,1 (janvier 1933): 23.

73. Ibid. 
requises avec notamment l'arrivée de l'infirmière-hygiéniste. Il faut par exemple rehausser et uniformiser les critères de sélection entre les écoles d'infirmières. Mais il semble insuffisant de se limiter à une réforme éducationnelle. On parle d'une action conjointe entre les instances gouvernementales et les associations d'infirmières diplômées pour réorganiser socialement et administrativement la profession. La clef de voûte de cette réorganisation serait la mise sur pied d'un système de classification des infirmières diplômées afin de préconiser «une orientation systématique des étudiantes vers les nouvelles carrières $^{74} \gg$ : le service d'État, le service institutionnel, le service d'hygiène publique et le service éducationnel.

Forte de la légitimité que concède ce rapport aux infirmières dans leur volonté d'autonomie, la présidente du registre Ville-Marie de l'Association canadienne des infirmières catholiques, Annonciade Martineau $^{75}$, va jusqu'à revendiquer le contrôle total de la revue par les infirmières: "Prenons conscience de nos responsabilités [...] et peut-être qu'à son $25^{\mathrm{e}}$ anniversaire, la revue, suivant la loi de la nature, sera rédigée et dirigée par les gardes-malades ${ }^{76} . »$ Et d'ajouter: «Qui connaît mieux les intérêts de notre profession, que nous, les intéressées ${ }^{77} . »$

En mars 1939, Charlotte Tassé commente les propos tenus par le docteur H. B. Atlee ${ }^{78}$ lors d'un congrès national d'infirmières à Halifax. En substance, ce dernier croit que la profession infirmière est tenue en état d'infériorité et il s'interroge sur les raisons de cette situation:

Pourquoi votre travail, pourtant si important, n'a-t-il pas été reconnu à son mérite? Pourquoi les hôpitaux ne sollicitent-ils pas votre avis et vos conseils? Pourquoi n'avez-vous pas votre part dans les organismes qui s'occupent de la santé publique? Pourquoi, telles des soldats, vous soumet-on à des règlements plus

74. Ibid., 25 .

75. Ce registre est instauré en janvier 1935 dans le but de centraliser les appels, de fixer une échelle de prix, de faire de la publicité, d'offrir des services à l'heure, etc. Pour une note biographique plus complète, voir Yolande Cohen et Michèle Gélinas, «Les infirmières hygiénistes de la ville de Montréal: du service privé au service civique», Histoire sociale/Social History, 22,44 (novembre 1989): 219-246.

76. Annonciade Martineau, «Dix ans... Infirmières, Réfléchissons», La GMCF, 10,1 (janvier 1937): 19.

77. Ibid.

78. Le docteur Atlee est professeur de gynécologie et d'obstétrique à l'université de Dalhousie. Sa conférence a été publiée par The Canadian Nurse. 
sévères que ceux qui régissent les autres professions? Pourquoi vous exploite-t-on comme des gens inférieurs ${ }^{79}$ ?

Après avoir rendu hommage à la profession médicale, Charlotte Tassé demande aux infirmières «de lire et relire cet article, de le méditer longuement afin qu'elles voient bien le long chemin que nous avons à parcourir pour atteindre le but rêvé par le docteur Atlee ${ }^{80}$ ». Son appel est entendu par des infirmières laïques, de plus en plus conscientes de l'importance de réfléchir à leur statut professionnel, et qui collaborent plus activement à la revue dans les années $1940^{81}$. Le pouvoir décerné à la sœur hospitalière est désormais la cible des critiques. Une infirmière qui conserve l'anonymat demande, dans la rubrique Le Courrier de Djénane ${ }^{82}$, quel rang doit occuper la sœur hospitalière. Elle obtient la réponse suivante:

[...] la religieuse en entraînement doit être considérée comme l'infirmière tout en tenant compte du respect dû à son état religieux. Il est entendu que la religieuse, comme l'infirmière lä̈que, doit faire partie des associations, si elle a rempli les conditions pour y appartenir [...] Quant à confier à la religieuse un poste actif, de premier plan, c'est plus difficile à cause du règlement, et non de sa compétence. [...] la religieuse ne vivant pas dans le monde peut difficilement apporter certaines solutions logiques aux problèmes qui surgissent en grand nombre dans les associations et qui sont d'ordre purement profane. Un rang honorifique est désigné plutôt pour la religieuse faisant partie des associations $^{83}$.

Les limites d'intervention de la sœur hospitalière au sein de la société laïque sont de plus en plus mises en évidence. La cassure s'effectue réellement en 1941. C'est à ce moment que La GMCF met en place une tribune libre. Cette innovation répondrait à certaines revendications des infirmières. La direction veut inciter les infirmières

79. Charlotte Tassé, «L'avenir de la profession», La GMCF, 12,3 (mars 1939): 103. Cet article comporte la traduction intégrale de la conférence du docteur H. B. Atlee, précédée d'une introduction par Charlotte Tassé. La direction de la revue emploie la forme italique pour mettre le passage cité en évidence.

80. Ibid.

81. Entre 1928 et 1940 , la proportion des articles écrits par des infirmières laïques a plus que triplé, passant de $20 \%$ en 1928 , à $72 \%$ en 1940 .

82. La rubrique apparaît en octobre 1940. Elle cesse par la suite, mais elle revient en 1947. La direction dit que cette rubrique a pour objectif «de discuter des problèmes que la vie fait inopinément surgir ici et là. Le tout est confidentiel et le pseudonyme est accepté pour voiler l'identité». «Le courrier de Djénane», La GMCF, 20,9 (septembre 1942): 394.

83. «Courrier de Djénane», La GMCF, 13,11 (novembre 1940): 513. 
à sortir de leur mutisme. Du moins, c'est ainsi que la première intervenante ${ }^{84}$ s'explique:

[...] les temps actuels ne se prêtent plus à un régime d'autoritédictature pour nos directives, d'où qu'elles viennent [...] En ouvrant ainsi une 'Tribune libre', nous échappons du même coup à la mise en tutelle et à l'étouffement de notre opinion libre, que nous avons le droit d'exprimer en toute occasion ${ }^{85}$.

Les commentaires des collaboratrices qui osent maintenant s'identifier sont plus incisifs, tel celui d'Anne-Marie Dutaud:

C'est bien parce que, dans le fond, les infirmières s'intéressent réellement aux questions qui les regardent, qu'elles ont réclamé depuis longtemps, d'exprimer leur opinion sur ce qui se passe, dans leur organe: «LA GARDE-MALADE CANADIENNEFRANÇAISE»; et cela dans le seul but d'améliorer la situation. [...] Nous avons accepté trop longtemps 'la consigne du silence et de l'obéissance' ${ }^{86}$.

Il est clair qu'une transformation majeure est en cours. Il n'est plus question de dévouement pour autrui mais bien d'autonomie et de prise en charge collective de son avenir. Par conséquent, en cherchant à se dégager du caractère confessionnel, du modèle traditionnel, il devient possible de caractériser davantage les soins infirmiers par de prétendues qualités féminines. Ainsi se met en place un modèle professionnel de type identitaire, qui s'accommode de la laïcité et de la science, mais qui ne veut aucunement rompre avec ce qui en a déterminé l'origine, la féminité et, bien sûr, l'ancrage national canadienfrançais. Car, si le modèle canadien-anglais est encore présent, il inspire aussi la revendication d'une distinction, l'émergence d'un modèle propre. À cet effet, Charlotte Tassé souligne à plusieurs reprises l'importance des spécificités nationales ${ }^{87}$.

On notera d'ailleurs que les perspectives d'émancipation des femmes n'intéressent guère la revue qui se cantonne à la défense d'une vision de la féminité et du maternage comme base des soins infirmiers, du care. $L a G M C F$ fait très peu référence aux autres femmes, celles qui pratiquent désormais la médecine ${ }^{88}$, cette chasse gardée de

84. Elle signe tout simplement Une infirmière canadienne-française.

85. «Tribune libre», La GMCF, 14,4 (avril 1941): 157.

86. Anne-Marie Dutaud, «Tribune libre», La GMCF, 14,10 (octobre 1941): 447.

87. Voir notamment Charlotte Tassé, «Affirmons-nous», $L a G M C F, 20,6$ (juin 1947).

88. On peut noter un article de Simonne Sélinas qui déplore les conditions de pratique de la femme-médecin. «Femmes d'aujourd'hui», La GMCF, 20,4 (avril 1947): 187-189. 
l'homme ${ }^{89}$ et reste sourde aux perspectives d'émancipation, par l'obtention du droit de vote par exemple. La conférence de Thaïs LacosteFrémont ${ }^{90}$, devant l'Association des infirmières de l'hôpital SainteJustine de Montréal, critique l'utilisation abusive du dévouement maternel par les politiciens et souhaite sa revalorisation:

Tant que les lois d'un pays considèrent la femme mariée comme un enfant, une mineure, une incapable, la société la traite ainsi et ne peut recevoir de la mère le plein rendement de sa personnalité féminine et de son dévouement maternel. Il en est encore ainsi chez nous dans cette province ${ }^{11}$.

La transformation du modèle proposé par la revue est donc largement réalisée. Elle est concommittante à l'essor et à la diversification de la pratique infirmière au Québec. La spécialisation accrue, le développement considérable du secteur public avec l'importance grandissante des institutions hospitalières sont autant d'éléments de ce changement. Les infirmières-hygiénistes, qui œuvrent dans des unités sanitaires de comté ou en service privée ${ }^{92}$, disposent d'une marge de manœuvre très grande, en ayant la responsabilité de la prévention, des visites à domicile, des conférences publiques et de l'inspection des écoles.

Quand éclate la Deuxième Guerre mondiale, la forte implication des infirmières dans le conflit et la pénurie des effectifs ${ }^{93}$ contribuent à mettre en évidence l'importance des infirmières et à accroître leur prestige. Amorcée au nom du développement de la science médicale et de la santé publique, mais aussi de l'ajustement au modèle préconisé par les organisations internationales d'infirmières, l'affirmation de leurs prérogatives n'est désormais plus contestable. Couronnant ces années d'activisme, les infirmières obtiennent l'adoption en 1946

89. Par exemple, une infirmière de Belgique, L. Vankeeberghen, préconise, au cours d'une séance plénière du Cinquième congrès mondial du comité international catholique des infirmières et assistantes médico-sociales, que l'infirmière «ne doit pas s'appliquer à devenir un demi-médecin». «Extrait de quelques conférences prononcées au $5^{e}$ Congrès mondial du CICIAMS», La GMCF, 27,9 (septembre 1954): 13.

90. Thaïs Lacoste-Frémont est la sœur de Justine Lacoste-Beaubien, présidente du Conseil d'administration de l'hôpital Sainte-Justine.

91. Thaïs Lacoste-Frémont, «La garde-malade au point de vue de la famille», La GMCF, 22,5 (mai 1949): 211.

92. En 1926 est fondée la première unité sanitaire et en 1939, il y en a 42 à travers la province. «Les unités sanitaires», La GMCF, 19,11 (novembre 1939): 593-594.

93. Encore en août 1949, on indique un besoin de 8200 infirmières dans les seuls hôpitaux canadiens. Les effets de la guerre sont accrus par la popularité croissante de l'infirmière hygiéniste. «Pénurie grave d'infirmières aux hôpitaux», La GMCF, 22,8 (août 1949): 373. 
d'une loi leur reconnaissant un champ de pratique semi-exclusif ${ }^{94}$. Cette loi oblige les infirmières à s'inscrire à une corporation fermée, l'Association des infirmières de la province de Québec, qui a paradoxalement pour présidente une religieuse, sœur Valérie de la Sagesse de l'hôpital Sainte-Justine.

Le conflit n'est plus entre religieuses et laïques, mais plutôt entre professionnelles et auxiliaires. Les infirmières montréalaises se syndiquent au cours de la même année en fondant l'Alliance des infirmières de Montréal, affiliée à la Confédération des travailleurs catholiques du Canada. Elles sont amenées à prendre conscience de leur statut de travailleuses salariées, réticentes à être des «missionnaires laïques ${ }^{95}$ ». L'héritage apostolique de la pratique infirmière se traduit désormais par l'affiliation à un syndicat catholique:

[...] nous pouvons nous demander s'il y a compréhension véritable chez ceux, trop nombreux, qui tentent de conserver, comme héritage à notre carrière une tradition de pauvreté et de don de soi-même, poussé jusqu'au sacrifice absolu; ce qui est d'un autre âge mais n'est plus en harmonie avec les exigences économiques et sociales des temps présents ${ }^{96}$.

Mais c'est là une autre histoire. En ce qui nous concerne, il reste à savoir si le nouveau modèle de Tassé et de ses consœurs est aussi celui des clercs et des médecins qui écrivent dans la revue.

\section{Le repli du clergé et des sœurs hospitalières}

Les articles signés par les membres du clergé n'ont jamais été très nombreux. Ils représentent $29 \%$ des articles signés durant la première année de parution, et tombent ensuite à $15 \%$ en 1936, à $14 \%$ en 1940 pour arriver finalement à $6 \%$ en 1949 . Toutefois, si les membres du clergé ne s'y expriment pas directement, on peut penser qu'ils influencent la direction de la revue d'une autre façon. Car, après l'éviction des sœurs hospitalières, qui d'autre défendra la conception chrétienne des soins infirmiers? Les membres du clergé seront-ils à leur tour écartés de la direction d'une revue qu'ils ont contribué à fonder?

94. Les infirmières pouvaient obtenir des certificats de spécialisation depuis 1944. Mais c'est en 1946 qu'elles disposent officiellement de la semi-exclusivité d'un champ de pratique à partager avec les aides et les auxiliaires. Voir Robert Germain, Le mouvement infirmier au Québec: 50 ans d'histoire (Montréal, Bellarmin, 1985).

95. Johanne Daigle, L'émergence et l'évolution de l'Alliance des infirmières de Montréal, 1946-1966, mémoire de maîtrise (histoire), Université du Québec à Montréal, 1983, 29.

96. Mlle C. Marinier, "L'infirmière n'est pas assez appréciée», La GMCF, 19,3 (mars 1946): $127-130$. 
$\mathrm{Au}$ cours des premières années de parution, $\mathrm{M}^{\mathrm{gr}}$ Piette réhabilite la conception apostolique de l'infirmière canadienne-française en faisant de Jeanne Mance «le plus imposant modèle de l'infirmière canadienne $^{97}{ }^{»}$. Elle possédait une âme d'héroïne et les qualités nécessaires à la pratique infirmière: patience, discrétion, abnégation, réserve, bonté, charité chrétienne. L'infirmière doit suivre la voie tracée par Jeanne Mance et être en tout temps «une demoiselle exemplaire ${ }^{98}$ », elle qui est parfois appelée à remplacer le prêtre et à pratiquer l'ondoiement dans des cas d'urgence ${ }^{99}$. Vingt ans plus tard, $\mathrm{M}^{\mathrm{gr}}$ Desranleau, évêque de Sherbrooke, dans un discours prononcé lors du Cinquième congrès annuel provincial des infirmières du Québec, les invite à la prudence:

On se croit capable de tout lire, de tout voir, d'essayer tout, de goûter à tout... Non, les gardes-malades ne doivent pas tout lire. Il y a une foule de livres qu'on ne peut, qu'on ne doit pas lire. Vous ne pouvez pas tout voir non plus [...] Il faut toujours avoir les yeux tournés vers Rome; c'est le Pape qui est chargé de nous conduire jusqu'au ciel. Gardez bien l'esprit de votre association catholique; vous serez avec le Pape, avec l'Église et, de cette façon, vous êtes sûres du succès ${ }^{100}$.

L'importance d'une bonne formation technique ne doit pas faire oublier que, selon le père Garesche, «les valeurs spirituelles ont le pas sur les valeurs matérielles, ainsi l'instruction religieuse de l'infirmière a une importance plus grande que sa formation professionnelle ${ }^{101} \gg$. Il y a lieu de revaloriser la doctrine chrétienne au point de faire de l'hôpital un centre d'instruction catéchistique.

Les infirmières sont considérées encore par le clergé comme le fer de lance de l'apostolat catholique dans le secteur de la santé. Contestant l'intervention accrue de l'Etat dans ce domaine, le clergé cherche à sauver les meubles. Le père Émile Bouvier ${ }^{102}$ critique en février 1945 l'avant-projet de loi de l'assurance-maladie qui, même s'il lui apparaît acceptable dans son ensemble, démontre l'accaparement du

97. $\mathrm{M}^{\mathrm{gr}}$ Piette, «Lettre de $\mathrm{M}^{\mathrm{gr}}$ A.-V.-J. Piette», $L a G M C F, 1,2$ (février 1928): 34-36. On dit de la fondatrice de l'Hôtel-Dieu de Montréal qu'elle fut la première infirmière laïque de l'Amérique du Nord.

98. Père A.-H. Crevier, «La morale professionnelle de la garde-malade», $L a G M C F, 2,8$ (août 1929): 354-355.

99. Abbé C.-Philippe Normand, «Échos et Communications», La GMCF, 2,11 (novembre 1929): 531 .

100. $\mathrm{M}^{\mathrm{gr}}$ Desranleau, «À travers les journaux», $L a$ GMCF, 21,11 (novembre 1948): 521.

101. Père Garesche, «La formation religieuse des infirmières», $L a G M C F, 10,12$ (décembre 1937): 547 .

102. Il est à ce moment directeur de la section des relations industrielles de l'Université de Montréal et aviseur du Conseil des hôpitaux catholiques du Canada. 
social par l'État et le caractère universel du programme. Cela provoquera «la dissolution de toutes les organisations privées d'assurancesanté et [...] étouffera toute initiative volontaire de coopération dans le

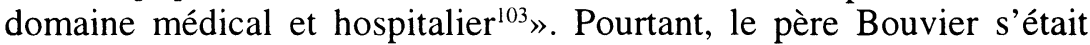
penché sur ces questions en 1940 en définissant ce que devait être le service social ou le samaritanisme moderne. D'après lui, «la société est une institution juridique, non un établissement de charité; elle est fondée sur le droit et le devoir et non simplement sur l'abnégation et le détachement ${ }^{104}$ ». Le clergé mène en quelque sorte un combat d'arrière-garde dans un domaine qu'il a déjà partiellement abandonné.

Les sœurs hospitalières se retrouvent ainsi relativement isolées. Leur collaboration à $L a G M C F$ se limite à faire l'histoire des premières écoles d'infirmières. Cette participation a pour but d'affirmer le rôle prépondérant qu'elles occupent à la direction de ces écoles, même si elles ne comptent plus que pour $11,5 \%$ des infirmières enregistrées $^{105}$. Leur vision de l'infirmière reflète cette position de domination des religieuses sur les infirmières laïques, mais aussi de subordination: l'infirmière est avant tout une femme qui demeure au service de l'homme comme l'infirmière est au service du médecin ${ }^{106}$. Pour sœur Bouffard, directrice des gardes-malades à l'hôpital Saint-Vincent-dePaul de Sherbrooke, même lors de cas douteux, l'infirmière se doit d'agir «tout en sauvegardant la réputation du médecin traitant ${ }^{107}$ ». Elles cherchent par ailleurs à étendre le champ de leur intervention dans des domaines où les infirmières laïques ont acquis une solide expertise. Sœur Francis mentionne notamment en octobre 1937 que «la pratique de l'obstétrique n'est pas pour des Religieuses incompatible avec la dignité et les exigences de leur état ${ }^{108}{ }_{\text {». }}$.

Étant donné l'influence encore prépondérante des sœurs hospitalières dans les écoles qu'elles gèrent et au sein des institutions hospitalières dont elles sont les propriétaires, leur mutisme étonne. Peut-on expliquer cette démission des sœurs sur le plan idéologique par l'in-

103. Père Émile Bouvier, «L'avant-projet de loi d'assurance santé», $L a G M C F, 28,2$ (février 1945): 81 .

104. Père Émile Bouvier, Le samaritanisme moderne ou Service social (Montréal, L'École sociale populaire, coll. «L'École sociale populaire», n 317, 1940), 4.

105. En mars 1931, E. Frances Upton fait état du taux d'enregistrement des infirmières depuis l'incorporation de l'association en 1920. Il y a eu 5350 infirmières qui ont obtenu leur diplôme comparativement à 615 sœurs hospitalières. E. Frances Upton, «L'enregistrement des gardes-malades diplômées», La GMCF, 4,3 (mars 1931): 118.

106. Sœur Saint-Ferdinand, «Les droits de la femme», $L a G M C F, 18,2$ (février 1945): 55.

107. Sour Bouffard, «Réponses aux questions posées par l'Université de Montréal aux candidates au diplôme d'infirmière universitaire», $L a G M C F, 7,8$ (août 1934): 461.

108. Sœur Francis, «Pourquoi les religieuses doivent pouvoir être sages-femmes», $L a$ GMCF , 10,10 (octobre 1937): 458-463. 
tervention de l'État ou par un abandon des communautés par l'Église, comme l'écrivent Nicole Laurin et Danielle Juteau ${ }^{109}$ pour caractériser l'intervention de l'Église et de l'État dans le secteur de la santé dans les années 1960? Repli stratégique caractérisant une volonté d'efficacité ou retraite obligée d'un milieu que l'on sent hostile, cette situation témoigne en tout cas dès les années 1940 du retrait complet des sœurs de la revue, et d'une transformation profonde de cette dernière. Les médecins sauront-ils relayer les aspirations apostoliques du clergé et voudront-ils préserver une conception chrétienne et apostolique des soins infirmiers?

\section{Les médecins et la défense d'une collaboratrice professionnelle}

En fait, au départ, les intervenants autorisés du clergé et des sœurs hospitalières font cause commune avec les médecins. La présence de ces derniers dans les pages de la revue est massive. À titre indicatif, les médecins sont les auteurs en 1928 de 47 des 92 articles signés (51\%) comparativement aux membres du clergé qui eux sont responsables de 27 articles signés (29\%). Encore en 1949, 20 des 52 articles signés le sont par des médecins $(45 \%)$. Très vite pourtant on voit se dessiner là aussi des changements d'alliance, l'objectif étant pour eux de renforcer leur position en éliminant progressivement la relation triangulaire religieuses/médecins/infirmières au profit d'une dynamique médecins/infirmières, bénie en partie par les membres du clergé.

Effectivement, les médecins se font d'abord les défenseurs d'un statu quo que ne renieraient pas les membres du clergé. Ils transmettent de nombreuses informations de nature médicale mais collaborent également à des articles de fond. On tient à un modèle apostolique de l'infirmière qui juxtapose les notions de science et de charité. L'infirmière doit être une collaboratrice scientifique obéissante en plus d'être empreinte de l'indispensable esprit de dévotion. Le médecin en charge du sanatorium Prévost, le docteur Edgar Langlois déclare en 1929: «Dans le rôle de la garde-malade comme dans celui du médecin [...] il y a un côté vraiment sacerdotal ${ }^{110}$.» Il met en garde contre tout abus de réglementation qui «mettrait une borne au dévouement et qui abaisserait au niveau d'un emploi quelconque leur belle profession qui n'a vraiment d'égale que la vie religieuse ${ }^{111} »$.

109. Nicole Laurin et Danielle Juteau, «La sécularisation et l'étatisation du secteur hospitalier du Québec, 1960-1966», dans Robert Comeau, dir., Jean Lesage et l'éveil de la nation (Sillery, Presses de l'Université du Québec, 1990).

110. Edgar Langlois, «Au Sanatorium Prévost», La GMCF, 2,10 (octobre 1929): 476.

111. Ibid. 
Cette image de la «missionnaire laïque» est largement partagée par ses confrères. Le docteur Léo Parizeau, radiologiste à l'Hôtel-Dieu de Montréal, souligne à l'intention des lectrices de La GMCF l'importance de l'appellation garde-malade. Qualifiant de mode française l'emploi du vocable infirmière, il soutient que le métier d'infirmière se caractérise d'abord par le soin aux malades. Il revient ainsi à la garde-malade de garder, de surveiller les malades pour les protéger et leur apporter aide et secours, sous les ordres du médecin:

Au risque d'offenser les susceptibilités de 3 ou 4 d'entre vous qui sont trop sujettes à sortir de leur rôle, je dirai que c'est à moi, médecin, d'indiquer l'ensemble des moyens qui vont guérir le patient; d'autre part, c'est à vous Mesdemoiselles, qu'il appartient de garder notre malade, c'est-à-dire de le protéger contre les sources d'infection et les visites importunes; à vous de l'aider de mille façons; à vous de le secourir si quelque danger subit le menace ${ }^{112}$.

Il s'agit de bien circonscrire la marge de manœuvre de l'infirmière et de limiter ses gains professionnels: "Que voulez-vous de plus, aimables lectrices? Voilà un vocable réservé à votre sexe; il reconnaît que vos activités sont d'un ordre professionnel et il vous concède même ce privilège de soigner que je voulais réserver aux seuls médecins $^{113} . »$

Dans l'immédiat, la publication du rapport Weir ne semble rien changer à cette situation. Au contraire, les médecins ressentent le besoin de rappeler les devoirs sacrés de l'infirmière envers le médecin. Le docteur Gérald Brisson s'adresse en septembre 1935 aux graduées de l'hôpital de la Providence à Montréal-Est. Il précise que l'infirmière manque tout simplement à son devoir «lorsqu'elle ne cultive pas la soumission et le respect au médecin ${ }^{114} \gg$. Cependant, les médecins sont conscients de l'autonomie que s'approprient des factions importantes de la profession, en particulier sous l'impulsion du mouvement hygiéniste ${ }^{115}$. Un haut niveau d'éducation est donc souhaitable. Par exemple, le docteur Adrien Plouffe entretient en 1936 les lectrices de $L a G M C F$ à propos de l'infirmière d'hygiène sociale:

112. Léo Parizeau, “'Infirmières' ou 'Gardes-Malades'», $L a G M C F, 1,5$ (mai 1928): 6.

113. Ibid., 7.

114. Gérald Brisson, «Échos et Communications», La GMCF, 9,1 (janvier 1936): 39.

115. Il faut mentionner que la profession médicale ne semble pas adopter un discours uniforme. Pendant que certains médecins cherchent à brimer la volonté d'autonomie du corps infirmier, d'autres se font plus conciliants et perçoivent les avantages pouvant découler d'une professionnalisation poussée de l'infirmière tout en demeurant les seuls maîtres à bord. 
La garde-malade canadienne-française tient-elle à rester dans son modeste coin ou tient-elle à se diriger vers les sommets? A-t-elle l'ambition de devenir quelqu'un ou se contenter d'une médiocrité qui, par ce temps de crise, ne saurait guère être dorée? Veut-elle être une force dans sa profession ou une petite bonne femme sans idéal et en somme peu intéressante? Veut-elle être la femme forte qui en impose par sa personnalité ou la pauvre jeune fille sans culture et sans originalité ${ }^{116}$ ?

Certains médecins comme le docteur Benoit critiquent ouvertement le modèle de l'infirmière catholique traditionnelle. Il insiste sur la nécessité de critères de sélection professionnels et non plus personnels afin que l'infirmière devienne le bras droit du médecin: «Quand une garde-malade entre dans une famille, c'est pour avoir soin d'un malade, ce n'est pas pour être cuisinière ou femme de chambre ${ }^{117}$.» En mars 1928, Benoit cherche à améliorer l'attitude du public envers les infirmières. Il prend la défense des infirmières à qui le public reproche essentiellement de coûter cher, en demandant une rémunération trop élevée, de se limiter à leur service et de se réserver des heures de repos et de loisir ${ }^{118}$. Il critique au passage les sœurs de la Charité de Québec qui souhaitent créer une école destinée à former des aides, à mi-chemin entre l'infirmière et la servante. Il s'insurge contre cette idée et demande de ne pas mettre aux côtés des écoles régulières, des écoles inférieures «abritées par le pavillon de la charité publique ${ }^{119}$ ». Le docteur Paul-H. Crevier soulève les mêmes problèmes dix-huit ans plus tard: «[...] les critiques sur la garde-malade proviennent de la confusion pratiquement générale [...] au sujet de la distinction entre gardes-malades diplômées et aides entraînées ${ }^{120}$.» D'après lui, une loi permettant de former une profession fermée est nécessaire. Les médecins tiennent à ce que les écoles d'infirmières soient mieux régies. Ils insistent sur l'importance de l'affiliation entre les écoles d'infirmières et l'Université de Montréal. Cette affiliation leur confère un plus grand contrôle sur la profession infirmière ${ }^{121}$. Charlotte Tassé réitère en 1940 son approbation à l'affiliation, alors que certaines infirmières questionnent sa pertinence ${ }^{122}$.

116. Adrien Plouffe, «L'infirmière et l'hygiène sociale», $L a G M C F, 9,6$ (juin 1936): 266.

117. E.-P. Benoit, «Les gardes-malades et le public», La GMCF, 1,3 (mars 1928): 68.

118. Ibid., 65 .

119. Ibid., 69.

120. Paul-H. Crevier, «Infirmière appréciée ou méconnue du public», La GMCF, 19,4 (avril 1946): 170.

121. Dès la première parution de $L a G M C F$, le docteur Benoit s'était efforcé de justifier le contrôle exercé par les médecins sur l'examen menant à l'obtention du titre d'infirmière diplômée. «L'université et la profession», $L a G M C F, 1,1$ (janvier 1928).

122. Charlotte Tassé, «Nos examens universitaires», La GMCF, 13,6 (juin 1940): 247-248. 
Ces considérations n'empêchent pas les médecins de revenir sans cesse sur le caractère féminin de la pratique infirmière. Une fois dégagés de l'emprise des sœurs hospitalières, ils peuvent en toute quiétude contrôler le degré de professionnalisme acceptable pour les infirmières et se faire les plus vigilants gardiens de la mission sacrée des femmes. Les médecins sont assez proches de Charlotte Tassé et des infirmières qui s'expriment dans la revue: débarrassée du contrôle des communautés religieuses et du travail domestique, l'infirmière éduquée pourra devenir l'adjointe d'autant plus éclairée du médecin que c'est lui qui s'est approprié sa formation. Jouant sur plusieurs tableaux, celui de la compétence et du dévouement, et sans s'émouvoir des contradictions et des conflits qu'ils peuvent générer, les médecins participent donc à l'élaboration du modèle d'une profession à laquelle ils sont liés par un intérêt professionnel commun (considéré auparavant comme un apostolat), mais qu'ils considèrent comme subordonnée (relations hommes-femmes mais aussi relations de savoir et de pouvoir) et à laquelle ils doivent concéder une certaine autonomie pour assurer l'efficacité des soins. Si ces conceptions rejoignent celles des infirmières de la direction de la revue, pour ce qui est de définir un modèle, elles ne seront pas nécessairement aussi bien accueillies dans la pratique.

\section{Une concurrence accrue avec The Canadian Nurse}

$\mathrm{Si}$ tous ces discours témoignent de la difficulté d'élaborer un modèle qui tienne compte des intérêts et perspectives de l'ensemble des intervenants de la revue, on voit bien malgré tout le clivage qui est en cours dès la fin des années 1940. Les médecins et infirmières laïques dominent l'espace de diffusion, reléguant le clergé et les sœurs hospitalières à la portion congrue. Il n'y a désormais plus d'obstacles à rejoindre le monde anglo-protestant, triomphant. Les problèmes de diffusion révèlent cette orientation. Il semble bien que $L a G M C F$ ne réponde plus aux besoins des infirmières laïques diplômées canadiennes-françaises, qui préfèrent s'abonner au périodique canadien-anglais The Canadian Nurse. Déjà en janvier 1932, à l'assemblée annuelle du comité général d'administration de $L a G M C F$, on avait discuté de la pertinence d'insérer une page en anglais, dans une optique de compétition $^{123}$.

123. L'origine de cette demande n'est pas mentionnée et on se contente de préciser: «L'assemblée ne se montrant pas favorable à la suggestion l'on délaissa le projet». "Assemblée annuelle de 'La Garde-Malade Canadienne-Française'», La GMCF, 5,2 (février 1932): 86. 
La direction souligne également, en novembre 1935, les efforts en vue d'augmenter le tirage de la revue et le nombre d'abonnements ${ }^{124}$. La revue est distribuée gratuitement dans les bureaux provinciaux à travers le Canada, et toutes les surintendantes d'associations d'infirmières canadiennes et américaines bénéficient du même privilège ${ }^{125}$. La situation semble s'améliorer à la veille des années 1940: «Depuis le mois de février 1939, la circulation a augmenté de beaucoup, [...] nous avons pu inscrire cent cinquante noms à notre tableau d'abonnement ${ }^{126}$.» La directrice est heureuse de constater en septembre 1942, lorsqu'elle trace le bilan des quinze années d'existence de la revue, qu'il y a «des articles pour trois mois d'avance ${ }^{127}$ » et qu'il y a par le fait même «trois fois plus de collaboratrices ${ }^{128}$ ».

Mais la situation se détériore de nouveau à la fin des années 1940 et au début des années 1950 . Tassé réagit en décidant de rejeter définitivement l'utilisation du vocable garde-malade. De plus, elle réfute tout lien qui pourrait subsister entre La Veilleuse et La GMCF: «[...] aucune relation n'a existé entre ces deux publications ${ }^{129} . »$ Elle renie en quelque sorte le fait qu'elle fut elle-même recrutée par les sœurs grises. La concurrence exercée par The Canadian Nurse, qui fait paraître une version française mensuelle à partir de 1958, devient vite insoutenable.

Tassé doit changer de cap: «En franchissant cette étape que nous croyons nécessaire à la diffusion de notre périodique, il me semble que nous nous séparons d'un passé difficile et parfois douloureux, mais auquel nous liaient de profondes racines ${ }^{130} . »$ Elle ajoute: «[...] nous avons compris que si nous voulions marcher avec le progrès, évoluer avec le nursing moderne, il fallait, de toute nécessité changer le nom de notre revue ${ }^{131}$.» La GMCF devient en 1957 Les Cahiers du nursing canadien. En récusant son passé canadien-français, la revue abandonne toute prétention à l'élaboration d'un modèle propre d'infirmière. L'emploi du terme anglais nursing symbolise l'adoption du modèle professionnel largement développé par les graduate nurses canadiennes-anglaises. Ainsi s'achève un épisode marquant de la ba-

124. La direction compte doubler le nombre d'abonnés.

125. Charlotte Tassé, «À l'aurore de 1939», La GMCF, 12,1 (janvier 1939): 8.

126. Charlotte Tassé, «Remerciements», La GMCF, 12,5 (mai 1939): 300.

127. Charlotte Tassé, «La Garde-Malade à quinze ans!», La GMCF, 15,9 (septembre 1942): 393

128. Ibid.

129. Charlotte Tassé, «Mise au point», La GMCF, 22,4 (avril 1949): 21.

130. Charlotte Tassé, «Message... votre revue change de nom», La GMCF, 29,12 (décembre 1956): 9 .

131. Ibid., 9. 
taille menée par une petite élite d'infirmières en faveur de l'élaboration d'un modèle identitaire de soins infirmiers. Il est significatif que cet abandon du modèle identitaire des soins infirmiers (canadien-français et apostolique) soit concomitant de l'abandon du rôle propre aux femmes, puisqu'à la même époque est abrogée l'exclusivité de la profession pour les femmes, ouvrant théoriquement la profession aux hommes.

\section{CONCLUSION}

Cette analyse montre l'étonnante longévité et la relative flexibilité d'un modèle apostolique, voué à une mort certaine par la modernité scientifique. La parution de La Veilleuse et de La GMCF atteste la volonté de constituer une nouvelle option cohérente, en réponse à la conception canadienne-anglaise de l'infirmière professionnelle. Les sœurs Duckett et Fafard se sont révélées capables de modifier, en l'améliorant, une conception des soins qui rendaient les infirmières attentives aux patients, tout en allant chercher une formation scientifique de premier plan. L'étonnant syncrétisme qui conduit progressivement à l'élaboration d'un modèle d'infirmière laïque conforme à la doctrine sociale de l'Église est remarquable. Ni le modèle de l'infirmière républicaine laïque ni celui de l'infirmière canadienne-anglaise, pourtant si proche, ne peuvent servir de référence exacte pour décrire le modèle de professionnalisation de l'infirmière canadienne-française. Les deux revues dédieront l'essentiel de leurs efforts à construire un modèle unique au Québec, soucieux de préserver la fonction apostolique de l'infirmière tout en convenant de la nécessité de sa formation professionnelle. Il faut voir dans les récents retours au care les reliquats de cette histoire.

Ce qui explique la persistance de ce modèle et son succès relatif, alors que l'ensemble du Canada se dote d'un corps d'infirmières professionnelles très organisées et assez autonomes dès le début du siècle, c'est l'intérêt commun qu'y voient médecins, membres du clergé et sœurs hospitalières. Ces dernières se saisissent de l'idéal professionnel pour parfaire la formation de leurs membres et s'assurer du maintien de leur contrôle sur l'accès à la profession. La mise en place d'un modèle identitaire sert à maintenir les autres, nouvelles venues et laïques, dans leur orbite. De cette façon, elles prolongent efficacement leur emprise sur la profession, plutôt que de s'en faire expulser, comme ce fut le cas en France ${ }^{132}$. L'attachement aux qualités fémini-

132. Voir Yvonne Knibiehler, dir., Cornettes et blouses blanches. Les infirmières dans la société française (1880-1980) (Paris, Hachette, 1984) et Véronique Leroux-Hugon, Des saintes laïques, les infirmières à l'aube de la Troisième République (Paris, Sciences en situation, 1992). 
nes a continué de fonder un modèle où dominent encore largement les femmes.

Mais l'analyse de contenu de ces deux revues montre bien aussi comment les positions respectives des sœurs hospitalières et des infirmières laïques furent bousculées au profit de la position dominante des membres du clergé et surtout de celle des médecins. Ces revues furent ainsi des outils de propagande idéologique entre les mains de quelques personnes plutôt que de véritables organes de promotion professionnelle.

Pour les médecins, même les plus éclairés, la perspective d'un modèle apostolique où ils tiennent le rôle dominant, juste après Dieu, a tout pour plaire. Les médecins parviennent dans l'espace de 30 ans à convaincre membres du clergé et sœurs hospitalières de leur faire confiance pour défendre les intérêts de l'Église dans le secteur de la santé et à assujettir les infirmières à leurs prérogatives professionnelles, au nom de la défense de la profession infirmière. La profession médicale réussit ce tour de force parce que l'Église est prête à faire alliance avec eux au détriment des communautés de religieuses hospitalières. Ces dernières se rabbateront pendant quelques années encore sur les écoles et la formation des infirmières, avant d'abandonner complètement le terrain aux laïques.

Ainsi, en continuant de faire la promotion d'un modèle désuet, les revues professionnelles des infirmières canadiennes-françaises ont réussi à masquer et peut-être aussi à retarder jusqu'à la veille de la Révolution tranquille, les changements profonds - la syndicalisation et la spécialisation des emplois dans le secteur hospitalier - que traversait la profession. 